\title{
ON The Peak FaCtor OF SAMPLed ANd Continuous Signals
}

\author{
Sergey Loyka \\ School of Information Technology and Engineering \\ University of Ottawa, 161 Louis Pasteur, Ottawa, \\ Ontario, Canada, K1N 6N5 \\ Email: sergey.loyka@ieee.org
}

\begin{abstract}
The peak factor of a continuous digitallymodulated signal is often analyzed from its samples taken at the Nyquist rate. This, however, may involve a significant error. It has been claimed, based on an illustrative example, that the peak factor of a continuous signal may be arbitrary large while the peak factor of the corresponding sampled signal is limited [10]. A validity of this example has been questioned in $[11,13]$ based on a flaw in [10]. In this paper, we demonstrate that the original illustrative example requires a small modification only to remove the flaw. It is also demonstrated that the continuous peak factor, in its traditional definition, may be arbitrary large while the sampled peak factor and the signal energy are bounded. An upper bound on the continuous peak factor of a BPSK sequence is derived.
\end{abstract}

Keywords: Peak-to-Average Power Ratio, Peak Factor, OFDM, sampling.

\section{Introduction}

Various types of OFDM systems became recently very popular for high-speed wireless communications, especially when the channel is frequency-selective [1,2]. While OFDM does offer significant advantages, it has a major drawback - the peak factor of an OFDM signal, sometimes refereed to as the peak-to-average power ratio, can be large [35], which is an obvious disadvantage from the RF amplifier view point. Thus, careful analysis of the peak factor is required. This problem has recently attracted significant attention and various peak factor reduction techniques have been proposed [5-9].

The peak factor of a continuous digitallymodulated signal is quite often analyzed using its samples at the Nyquist rate, i.e. no oversampling, due to convenience of such an approach. However, a certain difference exists between the peak factor of a continuous signal and its discrete counterpart. It has been recently demonstrated by Wulich, using an illustrative example, that the peak factors of sampled and continuous signal may be vastly different if no oversampling is used [10]. In particular, it was claimed that the continuous peak factor may be

\author{
Francois Gagnon \\ Department of Electrical Engineering, \\ Ecole de Technologie Superieure \\ 1100, Notre-Dame St. West, Montreal (Quebec), H3C \\ 1K3, Canada
}

arbitrarily large while the sampled peak factor is bounded. The validity of this example has been questioned in [11]. Specifically, it is claimed in [11] that the Wulich example is wrong because it has infinite derivative and a band-limited signal must have a finite derivative. However, this argument holds true provided that the band-limited signal itself is finite $[12$, p. $214-$ 215]. If the band-limited signal is infinite at some points, which is the case in the Wulich example, its derivative does not need to be finite. Thus, the claim in [11] is not justified.

A more detailed analysis of the Wulich's example and of some related issues has been given in [13]. In particular, it has been demonstrated that the continuous signal in the Wulich's example is infinite everywhere except for the sample points. Obviously, a signal with such a strange behavior, which is also impossible in practice, cannot be considered as a legitimate example. Also, it is not clear whether the Fourier transform and, consequently, the sampling theorem can be applied to such a signal because it does not satisfy the absolute integrability, or finite energy or finite power (over a finite time interval) conditions.

In this paper, we demonstrate that the original Wulich example requires a very small modification to meet the finite energy condition and, thus, the Fourier transform and the sampling theorem can be safely applied to the modified example. Also, the signal in the modified example is finite everywhere when the number of nonzero samples is finite. We also investigate the continuous peak factor of the modified signal as a function of the number of samples and provide a simple approximate expression for it and emphasize that this is the largest peak factor among any binary (BPSK) sequences of a given length, i.e. it provides an upper bound on the peak factors of any BPSK sequence. This bound is tight when the number of samples is large and it fits nicely into the bounds derived in [8] in a different context. Finally, we demonstrate that the continuous peak factor, as defined in [10] and many other papers, may be made arbitrary large while the sampled peak factor (without oversampling) and the signal energy are bounded. This example indicates that a certain amount of oversampling is essential for accurate estimation of the peak factor. 


\section{Modified Wulich Example}

The main idea of the modification of the original Wulich's example is to set the signal's samples equal to zero outside the observation interval $\left[-T_{o b s}, T_{o b s}\right]$ for which the peak factors are defined. We describe here this modification following [10]. The continuous-time signal in the Wulich example is given in terms of its samples:

$$
f(t)=\sum_{n=-\infty}^{n=\infty} f_{n} \frac{\sin \left[\omega_{M}(t-n T)\right]}{\left[\omega_{M}(t-n T)\right]}
$$

where $T=\pi / \omega_{M}$ is the sampling interval, $\omega_{M}$ is the maximum (radial) frequency in the signal's spectrum, and $f_{n}$ are the signal's samples. Note that this signal is strictly band-limited (since each term in (1) is bandlimited). The continuous peak factor is defined over the observation interval as

$$
P F_{c}\left(T_{o b s}\right)=\frac{\max _{-T_{o b s} \leq t \leq T_{o b s}}\left\{|f(t)|^{2}\right\}}{\frac{1}{2 T_{o b s}} \int_{-T_{o b s}}^{T_{o b s}} f^{2}(\tau) d \tau}
$$

and the sampled peak factor is defined as

$$
P F_{s}\left(N_{o b s}\right)=\frac{\max _{-N_{o b s} \leq n \leq N_{o b s}}\left\{\left|f_{n}\right|^{2}\right\}}{\frac{1}{2 N_{o b s}+1} \sum_{-N_{o b s}}^{N_{o b s}} f_{n}^{2}}
$$

where $N_{o b s}=T_{o b s} / T$, so that the discrete observation interval is $\left[-N_{o b s}, N_{o b s}\right]$. It should be emphasized that these definitions are not specifically tailored for our example but are widely used in the literature (e.g. [6-9]). The signal's samples are set as (the reason for this particular setting will be seen below - these are the "worst" possible samples in terms of the difference between the continuous and sampled peak factors):

$$
f_{n}=\left\{\begin{array}{cl}
1, & n=0 \\
-(-1)^{n}, & 0<n \leq N_{o b s} \\
(-1)^{n}, & -N_{o b s} \leq n<0 \\
0 & \text { otherwise }
\end{array}\right.
$$

With this modification and contrary to the original example [10], the continuous-time signal $f(t)$ meets the finite energy condition for any finite $N_{o b s}$ (its energey is given below by eq. 10). Thus, the Fourier transform and the sampling theorem can be safely applied (which was not the case with the original Wulich example [10], and which generated some debates on the issue $[11,13])$. It is straightforward to see that

$$
P F_{s}\left(N_{o b s}\right)=1
$$

It can be shown that, for $f_{n}$ given by (4), the maximum value of $f(t)$ is achieved at $t=T / 2$,

$$
\begin{aligned}
f(T / 2) & =\sum_{n=-N o b s}^{n=\text { Nobs }} f_{n} \frac{\sin \left[\omega_{M}(T / 2-n T)\right]}{\left[\omega_{M}(T / 2-n T)\right]} \\
& =\frac{2}{\pi}\left(\frac{1}{1+2 N_{o b s}}+\sum_{n=1}^{\text {Nobs }} \frac{1}{n-0.5}\right)
\end{aligned}
$$

(note that there was a mistake in [10, eq. 7], which is corrected above). Using (6), the continuous peak factor can be bounded from below as:

$$
\begin{aligned}
P F_{c}\left(T_{o b s}\right) & \geq \frac{|f(T / 2)|^{2}}{\frac{1}{2 T_{o b s}} \int_{-T_{o b s}}^{T_{o b s}} f^{2}(\tau) d \tau} \\
& \geq \frac{|f(T / 2)|^{2}}{1+\frac{1}{2 N_{o b s}}} \\
& =\frac{4}{\pi^{2}} \frac{2 N_{o b s}}{1+2 N_{o b s}}\left(\frac{1}{1+2 N_{o b s}}+\sum_{n=1}^{\text {Nobs }} \frac{1}{n-0.5}\right)^{2}
\end{aligned}
$$

The second inequality in (7) is due to the fact that

$$
\begin{aligned}
\frac{1}{2 T_{o b s}} \int_{-T_{o b s}}^{T_{o b s}} f^{2}(t) d t & \leq \frac{1}{2 T_{o b s}} \int_{-\infty}^{\infty} f^{2}(t) d t \\
& =\frac{T}{2 T_{o b s}} \sum_{n=-N_{o b s}}^{n=N_{o b s}} f_{n}^{2} \\
& =1+\frac{1}{2 N_{o b s}}
\end{aligned}
$$

Finally, using (7), we observe that,

$$
\lim _{T_{o b s} \rightarrow \infty} P F_{c}\left(T_{o b s}\right)=\infty
$$

(because of the $2^{\text {nd }}$ term in the last expression in (7) is divergent), i.e. the continuous peak factor can be made arbitrary large by increasing $N_{o b s}$ to a sufficiently large value (for fixed $T$ and, consequently, the fixed bandwidth), while the sampled peak factor is equal to unity (see (5)). This constitutes the modified Wulich example.

However, a few notes should be made regarding practical implications of this illustrative example. First, we note that in all practical systems $N_{o b s}$ is limited. Obviously, this limits the peak factor in (7) $\left(P F_{c}\right.$ is actually very close to the lower bound, as eq. (13) latter 
emphasizes). As it has been pointed out in [13], the level of a non-periodic signal and its variations are limited by the signal's energy [13, eq. (13)-(15)]. The energy of our modified signal is:

$$
E=\left(2 N_{o b s}+1\right) T
$$

Thus, when only finite-energy signals are considered, $N_{o b s}$ is limited as

$$
N_{o b s} \leq \frac{1}{2}\left(\frac{E}{T}-1\right)
$$

which obviously limits the continuous peak factor of the signal in (1).

However, we note that the continuous and sampled peak factors, as defined by eqs. (2) and (3) above, are invariant when a signal is multiplied by a constant, i.e. $f(t)$ and $f^{*}(t)=c \cdot f(t)$ have the same continuous and sampled peak factors. Hence, we define the constant $c$ as

$$
c=\frac{1}{\sqrt{\left(2 N_{o b s}+1\right) T}}
$$

With this definition, $f^{*}(t)$ has unit energy for any $N_{\text {obs }}$ (this is seen directly from (10)) and the same peak factors (continuous and sampled) as $f(t)$, i.e. given by (5) and (7). Thus, it demonstrates that the continuous peak factor, as defined by eq. (2), which seems to be a widely-accepted definition, may be arbitrary large (by increasing $N_{\text {obs }}$ to a sufficiently large value) while the sampled peak factor and the signal energy are bounded. It should be noted that, in this case, increase in $P F_{c}$ is accompanied by decrease in the average signal power. Hence, when thermal noise is considered, it may limit the usefulness of such an example for large $N_{o b s}$. While the practical implication of this modified example may be limited, it nevertheless demonstrates an interesting theoretical property of the widely-used peak factor definition.

The second practical implication of this example follows from the fact that for large $N_{o b s}$ the peak factor in (7) is very close to the lower bound, i.e. it can be approximated as:

$$
\begin{aligned}
P F_{c}\left(T_{o b s}\right) & \approx \frac{4}{\pi^{2}} \frac{2 N_{o b s}}{1+2 N_{o b s}}\left(\frac{1}{1+2 N_{o b s}}+\sum_{n=1}^{N o b s} \frac{1}{n-0.5}\right)^{2} \\
& \approx \frac{4}{\pi^{2}}\left(\ln \left(2 N_{o b s}\right)+1\right)^{2}
\end{aligned}
$$

where the $2^{\text {nd }}$ equality follows from the approximation of the sum by an integral [15]. Thus, the continuous peak factor is only logarithmic in $N_{o b s}$
, i.e. it increases very slowly with $N_{o b s}$ and, hence, assumes only moderately large values for any practical system. Fig. 1 compares $P F_{c}$ computed numerically with its approximation in (13), which appears to be reasonably accurate. The difference between $P F_{c}$ and the right-hand side of (7) is due to the limits of integration in (2), which are set to $\left[-T_{o b s}, T_{o b s}\right]$ rather than $[-\infty, \infty]$. If these limits are changed to $[-\infty, \infty]$, then the inequality in (7) becomes an equality.

It is straightforward to show (using conventional optimization techniques) that the sample sequence in (2) is the worst-case one in the sense that it results in the largest continuous peak factor among any binary sequence (BPSK) of a given length and amplitude. Thus, one may expect that any practical continuous peak factor of a binary sequence will not grow faster, i.e. (13) provides an upper bound. It is interesting to note that, despite of the fact that (13) has been derived for a BPSK signal, it is similar to the peak factor of coded OFDM signals in [6] and to the bounds of the peak factor of power-limited OFDM-modulated signals in [8], which also exhibit a square-logarithmic behavior.

\section{Acknowledgement}

The authors would like to thank J. Belzile for insightful comments. One of us (F. Gagnon) also thanks H. Minn for useful advices.

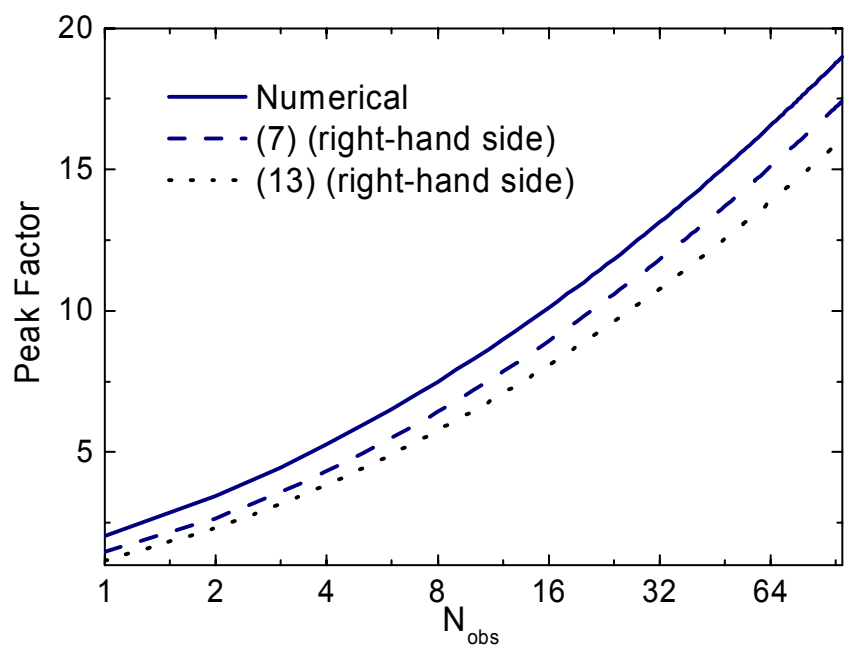

Fig. 1. Comparison of the numerically-computed peak factor with the approximate expressions. 


\section{References}

[1] M. Shafi et al, Wireless Communications in the 21st Century, IEEE Press and Wiley, 2002.

[2] G.L. Stuber et al, Broadband MIMO-OFDM wireless communications, Proceedings of the IEEE, v. 92, N. 2, pp. 271 - 294, Feb 2004.

[3] H. Ochiai, H. Imai, On the distribution of the peak-to-average power ratio in OFDM signals, IEEE Transactions on Communications, v. 49, N. 2, pp. $282-289$, Feb 2001.

[4] N. Dinur, D. Wulich, Peak-to-average power ratio in high-order OFDM, IEEE Transactions on Communications, v. 49, N. 6, pp. $1063-$ 1072, June 2001.

[5] S.H. Han, J.H. Lee, An overview of peak-toaverage power ratio reduction techniques for multicarrier transmission, IEEE Wireless Communications, v. 12 , N. 2 , pp. $56-65$, April 2005.

[6] K.G. Paterson, V. Tarokh, On the Existence and Construction of Good Codes with Low Peak-toAverage Power Ratios, IEEE Trans. Information Theory, v. 46, N. 6, pp. 1974-1987, Sep. 2000.

[7] C. Tellambura, Computation of the ContinuousTime PAR of an OFDM Signal with BPSK Subcarriers, IEEE Communications Letters, v. 5, N. 5, May 2001, pp. 185-187.
[8] S. Litsyn, A. Yudin, Discrete and continuous maxima in multicarrier communication, IEEE Trans. Information Theory, v. 51, N. 3, pp. $919-928$, March 2005.

[9] G. Wunder, H. Boche, Peak value estimation of bandlimited signals from their samples, noise enhancement, and a local characterization in the neighborhood of an extremum, IEEE Transactions on Signal Processing, v. 51, N. 3, pp. $771-780$, March 2003.

[10] D. Wulich, Comments on the peak factor of sampled and continuous signals, IEEE Communications Letters, v. 4, N. 7 , July 2000, pp. $213-214$

[11] N. Ermolova, Comments on "comments on the peak factor of sampled and continuous signals", IEEE Communications Letters, v. 5, N. 3 , March 2001, p.77

[12] J. Arsac, Fourier Transforms and the Theory of Distributions, Prentice-Hall, 1966.

[13] H. Minn et al, "On the Peak Factors of Sampled and Continuous Signals," IEEE Comm. Letters, v. 5, N. 4, pp. 129-131, April 2001.

[14] M. Sharif et al, On the peak-to-average power of OFDM signals based on oversampling, IEEE Transactions on Communications, v. 51, N. 1, pp. 72 - 78, Jan. 2003.

[15] Ya. B. Zel'dovich, A.D. Myshkis, Elements of Applied Mathematics, Moscow: Nauka, 1967 (in Russian). 